\author{
Jean-Michel Jeannin
}

\section{Geschichtlicher Überblick}

Älteste Zeugnisse für die Herstellung von Bier stammen aus dem 4. Jahrtausend v. Chr. von den Sumerern. Die im 2. Jahrtausend v. Chr. nachfolgenden Babylonier kannten schon 20 Biersorten, die sie aus Gerste, aus Emmer sowie aus verschiedenen Mischungen davon brauten. Die antiken Ägypter brauten ihr Bier aus Brotteig. Bei den antiken Griechen und den Römern galt das Bier hauptsächlich als Heilmittel. Von Cäsar (100-44 v. Chr.) ist allerdings überliefert, dass er seinen Gästen Bier in goldenen Bechern anbot. Im europäischen Mittelalter war das Bierbrauen ein Frauenberuf. Später übernahmen Mönche diese Aufgabe, zunächst für den Eigengebrauch, dann auch als Gewerbe. Zum Würzen des Bieres wurde lange Zeit die «Grut» verwendet, eine Mischung von verschiedenen Kräutern und anderen $\mathrm{Zu}$ taten, wozu je nach Braumeister auch Fichtenspäne, Kiefernwurzeln und Bilsenkraut verwendet wurden. Später ersetzte der Hopfen die Grut. Er verbessert die Haltbarkeit des Bieres und stabilisiert den Brauprozess. Das 1516 vom bayrischen Herzog Wilhelm IV. erlassene Reinheitsgebot legte fest, dass zur Herstellung von Bier nur Gerste, Hopfen und Wasser verwendet werden dürfen. Es gilt in Deutschland noch heute, wenn auch gelegentlich unter einem anderen Titel (Biersteuergesetz). Erst die Erforschung der Fermentation durch Louis Pasteur (1822-1895) und die Erfindung der Kältemaschine durch Carl von Linde (1842-1934) gegen Ende des 19. Jahrhunderts ermöglichten die ganzjährige kontrollierte

\title{
Genussmittel und Ganzheitsmedizin (2)
}

\section{Bier}

Herstellung von Qualitätsbieren, wie wir sie heute kennen [1].

\section{Hordeum distichum nutans $\mathrm{L}$. (Sommergerste)}

Bei der Gerste handelt es sich um eine Getreideart. Morphologisch zeichnet sie sich durch ihre langen Grannen aus. Die reifen Früchte werden sowohl als Nahrung als auch zum Bierbrauen verwendet. Die Gerste liefert 70-90\% der nachweisbaren Polyphenole des Bieres, darunter $(+)$-Catechin, Procyanidin B3, Procyanidin C2, Prodelphinidin B3 und Prodelphinidin C2 [2].

\section{Humulus lupulus L. (Cannabaceae)}

Der Hopfen (Abb. 1) ist eine einjährige bis ausdauernde Schlingpflanze aus der Familie der Hanfgewächse. Arzneilich und für das Bierbrauen werden die weiblichen Blütenstände verwendet. Der Hopfen verleiht dem Bier die beruhigende und den Appetit anregende Wirkung [3]. Er enthält reichlich Polyphenole, unter anderem Catechin-Gerbstoffe, Tannine, glykosidisch gebundenes Quercetin und Kaempferol, Catechine, Epicatechine, Proanthocyanidine, phenolische Carbonsäuren und das Chalkon Xanthohumol [4]. Der Hopfen liefert $10-30 \%$ der nachweisbaren Polyphenole des Bieres [2]. Weitere Inhaltsstoffe sind Humulone und Lupulone (Harzbitterstoffe) sowie ätherische Öle [3]. Es werden je nach Quercetinoder Kaempferol-Gehalt Bitterhopfen- und Aromahopfensorten unterschieden [4].

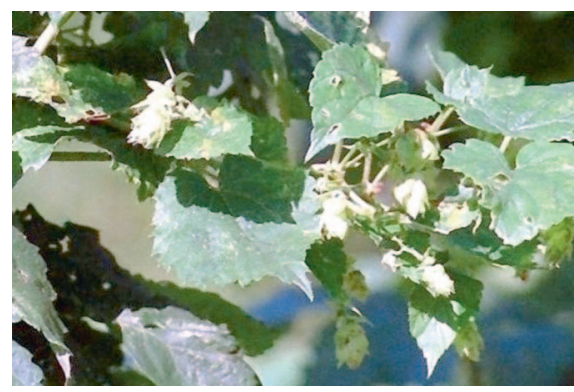

Abb. 1. Wilder Hopfen.

\section{Herstellung («Brauen») des Bieres}

Das Brauen von Bier ist ein komplexer Prozess, dessen Parameter sehr eng kontrolliert werden müssen, damit die gewünschte Qualität und Biersorte entstehen. Die Gerste wird durch Einweichen in Wasser zum Keimen gebracht und anschliessend getrocknet. Man erhält so das Malz, das geschrotet und dann mit Wasser gemischt wird (Maischen). Während dieses Vorgangs wird die Stärke der Gerste von Amylasen zu vergärbaren Zuckern abgebaut. Die Maische enthält nun die Inhaltsstoffe des Malzes, wird geläutert (filtriert) und unter Zugabe des Hopfens gekocht, geklärt und gekühlt. Man erhält eine geklärte Würze, die einer Gärung durch Hefe unterzogen wird. Es werden «obergärige» und «untergärige» Hefetypen unterschieden, die in unterschiedlichen Temperaturbereichen optimal arbeiten (15-20 bzw. 5-15 ${ }^{\circ} \mathrm{C}$ ). Obergärige Hefen schwimmen nach der Gärung auf dem Sud, untergärige Hefen setzen sich [5]. Die Wahl des Hefetyps beeinflusst den Charakter des Bieres. Nach der Gärung wird die Hefe abgetrennt, das Bier filtriert und dann abgefüllt [2]. Wird anstelle der Gerste Weizen verwendet, erhält man Weissbier.

\section{KARGER}

Fax +4976145207 14 Information@Karger.de www.karger.com
(C) 2012 S. Karger GmbH, Freiburg
Dipl. med. biol. Jean-Michel Jeannin Holeestrasse 43, 4054 Basel, Schweiz

Tel. +41 61-4215991, Fax -4230313

jmjeannin@datanetworks.ch 


\section{Chemie des Bieres}

Bier besteht aus Wasser, Stärke, Zucker und Ethanol. Das Ethanol liegt je nach Biersorte in unterschiedlicher Konzentration vor. Lagerbier z.B. enthält 3,9\%. Wichtige weitere Substanzen sind Polyphenole und Bitterstoffe [6]. Die Polyphenole stammen von der Gerste und dem Hopfen, von dem auch die Bitterstoffe stammen. Sie werden im Verlauf des Brauprozesses durch Kochen chemisch verändert. Ihre antioxidative Aktivität ist grösser als die des Weins [2]. Der Energiegehalt des Bieres wird gerne überschätzt, weil es den Appetit anregt und die Gewichtszunahme ihm selbst zugeschrieben wird. Er beträgt $159 \mathrm{~kJ} / 100 \mathrm{~g}$ - weniger als der von Apfel- oder Orangensaft (187 bzw. $194 \mathrm{~kJ} / 100 \mathrm{ml})$.

\section{Kulturelle Bedeutung}

Bier (Abb. 2) ist seit jeher mehr als nur ein Getränk. In den traditionellen Studentenverbindungen pflegt(e) man Rituale mit Biertrinken im Mittelpunkt. Seine heutige kulturelle Bedeutung hat das Bier aber erst durch die hohe Qualität und die breite $\mathrm{Pa}$ lette an Sorten erlangt, die erst durch die modernen Herstellungsmethoden

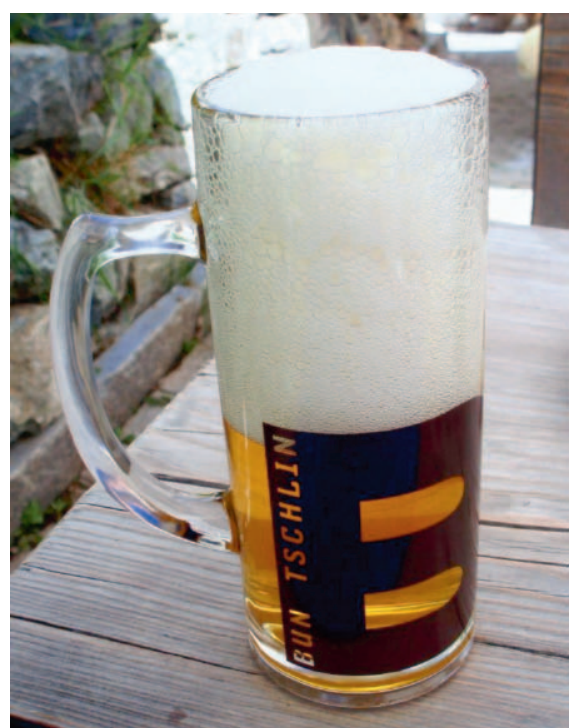

Abb. 2. Bier aus den Bergen (Tschlin, Engadin). ermöglicht wurden. Ein passendes Bier zu trinken, gehört zur gehobenen Esskultur. Da Bier Alkohol enthält, wird auch vor dem Biertrinken angestossen, und man wünscht sich Gesundheit. Das Anstossen stammt aus dem Mittelalter, wo so kräftig angestossen wurde, dass ein Austausch von Flüssigkeit zwischen den Bechern oder Krügen der Partner stattfand mit dem Risiko, dass man vom eigenen Gift zu trinken bekam, wenn man den Partner vergiften wollte [1].

\section{Traditionelle und volksmedizinische Verwendung als Arzneimittel}

Der berühmte Arzt Hippokrates von Kos (460-370 v. Chr.) schrieb dem Bier, das er als «Gerstensud» bezeichnete, eine Heilwirkung bei Fieber und Schlaflosigkeit zu. Cäsar versorgte seine Truppen mit Bier, das er für nahrhaft und kräftigend hielt. Hildegard von Bingen (1098-1179) empfahl schwermütigen Menschen Bier zur Hebung des Muts und zur Regeneration der Seele. Paracelsus (1493-1541) nannte das Bier eine göttliche Medizin. Zu seiner Zeit gab es Heilbiere, cerevisiae medicatae [2]. In der «Enzyklopädie der Volksmedizin» wird eine ganze Reihe von Anwendungen des Bieres als Hausmittel beschrieben. Bier und Zubereitungen aus Bier werden zur Behandlung der Wassersucht (Ödeme), zur Anregung des Milchflusses von Wöchnerinnen und zur Behandlung des Skorbuts empfohlen. Abgeraten wird «vollsaftigen» Personen der Konsum von Starkbieren, da dieser die Apoplexie begünstige [7].

\section{Gesundheitlicher Nutzen des Bieres}

Regelmässiger moderater Konsum alkoholischer Getränke, ob Wein, Bier oder Spirituosen, senkte die Inzidenz des Herzinfarkts in einer Studie aus Boston [8]. Die Autoren führen die Wirkung des Alkoholkonsums auf eine Senkung des Cholesterolspiegels sowie eine Erhöhung der HDL durch den Alkohol per se zurück. Truelsen et al. [9] fanden, dass mässiger Weinkonsum, nicht aber der Konsum von Bier und Spirituosen das Risiko eines Hirnschlags vermindern. Ob die Wirkung des Weins auf seinen Inhaltstoffen oder auf dem Umstand beruht, dass er meistens zu einer Mahlzeit eingenommen wird, konnten die Autoren nicht entscheiden. Dank seiner Bitterstoffe kann Bier zur Anregung des Appetits verwendet werden.

\section{Risiken}

Übermässiger Bierkonsum kann aufgrund des enthaltenen Alkohols zur Abhängigkeit führen. Bier berauscht, was die Suchtbildung fördert. Viele Unfälle werden vom Alkohol mindestens mitverursacht. Exzessiver Konsum kann akut gefährlich sein.

\section{Schlussfolgerung}

Bier enthält ausser Alkohol eine Reihe von pharmakologisch wirksamen Substanzen, die einen gewissen Gesundheitswert aufweisen. Geniesst man Bier mit Mass (bitte nicht verwechseln mit der «Bayrischen Mass»), hat man der Gesundheit eher genützt als geschadet.

\section{Literatur}

1 www.bier.de/bier-wissen/geschichtel geschichte-des-bieres.php.

2 Wollersen $\mathrm{H}$ : Bestimmung und Identifizierung von Flavonoiden in Gerste mit HPLCDAD-MS/MS. Dissertation. Paderborn, 2004.

3 Hopfen; in Pahlow M: Das grosse Buch der Heilpflanzen. Augsburg, Bechtermünz, 2000.

4 Kammhuber K: Quercetin und Kaempferol. Hopfenrundschau Int 2006/2007:52-55.

5 Schweizer Brauerei-Verband: Rohstoffe. www.bier.ch/deu/bier-brauen-rohstoffe.html.

6 Schweizer Brauerei-Verband: Inhaltsstoffe. www.bier.ch/deu/bier-inhaltsstoffe.html.

7 B: Bier, Cerevisia; in Most GF: Enzyklopädie der gesamten Volksmedizin. 1843. $w w w$. textlog.de/med-bier-cerevisia.html.

8 Gaziano JM: Type of alcoholic beverage and risk of myocardial infarction. Am J Cardiol 1999;83:52-57.

9 Truelsen $\mathrm{T}$, et al: Intake of beer, wine, and spirits and risk of stroke: the Copenhagen City Heart Study. Stroke 1998;29:2467-2472. 